Universitas, Volumen 1, Año 1, 2007,21-23

๑) 2007 UNAN-León, Editorial Universitaria

\title{
Aborto Inducido en Nicaragua
}

\author{
Arnoldo Toruño T.* \\ Departamento de Salud Pública. Facultad de Ciencias Médicas, Universidad Nacional Autónoma de Nicaragua, León. \\ (UNAN-León).
}

\section{RESUMEN}

En los países pobres, el aborto inducido en condiciones inseguras es un serio problema de salud pública. En Nicaragua, sabemos que ha ocupado entre el tercero y cuarto lugar entre las causas de mortalidad materna, pero se ha estudiado poco su frecuencia. Con esta finalidad, y con la de conocer algunas características de las mujeres que se lo practican, el tema fue incluido en tres encuestas sobre salud reproductiva, realizadas en 1997, 2001 y 2005, en cuatro departamentos: León, Chinandega, Managua y Rivas. En total fueron entrevistadas 16,511 mujeres entre los 15 y 49 años de edad, seleccionadas mediante muestreo por conglomerados. Se encontró una tasa de 1.3 abortos anuales por mil mujeres y una razón de 1.2 abortos por cien nacidos vivos. Con metodologías de encuestas específicas para este tema y sobre todo mediante el uso de estimaciones indirectas, cabe esperar cifras de frecuencia más altas. La frecuencia era mayor en la capital del país. Predomina en mujeres adultas, casadas o acompañadas y en mujeres que ya han tenido hijos. Abortaron, mujeres de todos los niveles educativos. Es importante notar que el número elevado de abortos inducidos ocurre a pesar de que la ley siempre los ha prohibido.

Palabras claves: Aborto inducido.

\section{INTRODUCCION}

En Nicaragua, la mortalidad materna es un problema altamente priorizado por el Plan Nacional de Salud, y el aborto ha sido una causa importante de esas defunciones. En efecto, en 1998, un estudio conjunto del Ministerio de Salud y UNICEF ${ }^{[1]}$, a la par de calcular una tasa de mortalidad materna de 133 por 100,000 nacidos vivos, señala al aborto como la tercera causa de muerte. Adicionalmente, reconoce el problema de subregistro de defunciones, mencionando entonces que en la realidad, la tasa podría ser de 200 por 100,000 nacidos vivos. En otra revisión más reciente, el aborto aparece como cuarta causa de muerte materna, pero señalando la posibilidad de que algunos casos hayan sido registrados como hemorragias o sepsis ${ }^{[2]}$.

La frecuencia del aborto inducido ha sido poco estudiada en Nicaragua, donde hasta Octubre del año pasado era permitido sólo por prescripción médica, y a partir de entonces, por ninguna causa. Un estudio realizado en 1993 en el Municipio de León, en el Occidente del país, mostraba que ocurría con una razón de 1.1 eventos por cada 100 nacidos vivos ${ }^{[3]}$.

De tal manera, se realizó el estudio con dos objetivos: determinar la frecuencia del aborto inducido, y conocer algunas características de las mujeres que abortan.

\section{DISEÑO METODOLOGICO}

En los años 1997, 2001 y 2005 se realizaron encuestas para conocer la situación de la salud reproductiva en cuatro departamentos de la zona del Pacífico de Nicaragua: Chinandega, León, Managua y Rivas. En total se entrevistaron 16,511 mujeres comprendidas entre los 15 y los 49 años de edad. El muestreo fue por conglomerados, tomando como tales los territorios correspondientes a las juntas receptoras de votos, que tienen un número de personas bastante similar entre sí. La probabilidad de selección de los conglomerados era proporcional a su tamaño.

En las dos primeras encuestas el muestreo fue en una etapa, entrevistando a todas las mujeres que quedaban en el conglomerado. En la tercera encuesta se usó muestreo en tres etapas, procediendo a una selección al azar de viviendas dentro de cada conglomerado, y a la selección de una mujer en la vivienda, mediante sorteo. En el cuestionario se incluyeron tres preguntas para conocer sobre la ocurrencia de abortos en los doce meses previos a la encuesta, y para saber si los mismos habían sido naturales o provocados. Las entrevistas se realizaban procurando un ambiente de privacidad. 


\section{RESULTADOS Y DISCUSION}

Entre las 16,511 mujeres entrevistadas, en los doce meses previos a la encuesta habían nacido vivos 1,784 niños y ocurrieron 243 abortos, de los cuales 217 fueron reportados como espontáneos, 22 como provocados, y en 4 casos las mujeres se negaron a reportar su naturaleza. En base a las cifras anteriores se obtiene una tasa de 1.3 abortos inducidos al año, por cada mil mujeres en edad fértil, y una razón de 1.23 abortos por cada 100 nacidos vivos.

Es muy probable que en el caso de las 4 mujeres que en nuestras encuestas se negaron a señalar la naturaleza de sus abortos, los mismos hayan sido inducidos, pues de otra manera no hubieran tenido reticencia para contestar. Tomándolos como tales, se llega a un total de 26 abortos inducidos, lo que significa una tasa de 1.57 abortos al año, y una razón de 1.46 abortos por cien nacidos vivos.

Al examinar nuestros resultados debemos tomar en cuenta la experiencia de Olinto y Moreira ${ }^{[4]}$, en Brasil, quienes usando cuestionarios autoadministrados y pidiendo a las mujeres que los depositaran en cajas cerradas, obtuvieron cifras de frecuencia de aborto que casi duplican las obtenidas mediante las encuestas convencionales, como las nuestras. Si esto fuera igual en Nicaragua, la cifra de 1.46 abortos por cien nacidos vivos se elevaría a 2.7 .

Por departamentos, la razón de abortos por 100 nacidos vivos fue la siguiente: a) León y Chinandega: 1.1 ; b) Managua: 1.5, у c) Rivas: 0.8. Cabe señalar que la cifra correspondiente a los departamentos de León y Chinandega es igual a la obtenida en el estudio en el Municipio de León, en 1993, citado anteriormente.

Cabe mencionar que nuestras cifras, obtenidas mediante encuestas, son bastante inferiores a las estimadas para el área centroamericana, a partir de métodos indirectos, bien sea a partir del análisis de los determinantes de la fecundidad en el modelo de Bongaarts, o a partir de datos de hospitalizaciones por complicaciones del aborto y de la proporción que éstas significan en relación al total de abortos inducidos.

En efecto, según la Organización Mundial de la Salud (OMS) ${ }^{[5]}$, en esta región ocurren 20 abortos inducidos por cada cien nacidos vivos, cifra muy parecida a la de 21 por cien, estimada por Henshaw y colaboradores ${ }^{[6]}$. Por su parte, Remez ${ }^{[7]}$ estima que México y República Dominicana, también en la región centroamericana, tienen respectivamente 39 y 21 abortos por 100 nacidos vivos.
En el año 2006 se calcula que nacieron vivos unos 141,200 niños. De acuerdo a los datos de nuestra encuesta, habrían ocurrido como mínimo unos 1,737 abortos inducidos. Si nos atenemos a las estimaciones de la OMS y de Hemshaw para el área centroamericana, ocurrieron unos 28,240 abortos de este tipo. De acuerdo a un estudio previo realizado en León por I. Gremy, M.L. Dussan y L. Gureau, la razón principal que tienen las mujeres para recurrir al aborto la constituyen las dificultades económicas, seguida de otras como el rechazo y las presiones familiares, el deseo de no tener un hijo de determinado hombre, el hecho de ser madre soltera y en menor medida, por problemas de salud. La mayoría de las razones antes expuestas nunca han sido permitidas por la ley nicaragüense.

Ahora que se revisa la legislación sobre el aborto, tanto en la Corte Suprema de Justicia como en la Asamblea, los datos anteriores deben ser tomados en cuenta.

Significan que las leyes restrictivas han mostrado escasa efectividad para impedir que ocurran los abortos en nuestro país. Por otra parte, el hecho de que el aborto inducido ocupe el tercero o el cuarto lugar entre las causas de mortalidad materna indica que seguramente buen número de mujeres se practican los abortos de manera clandestina, sin los procedimientos y condiciones adecuadas, lo que favorece las complicaciones y la muerte, tanto de ellas como del embrión o el feto que llevan en su vientre.

De tal manera, la lección clara es que ofrecer cárcel a las mujeres, lejos de ser una buena solución al problema, lo incrementa en términos de muertes maternas. Por lo tanto, se hace necesario buscar mejores alternativas de solución.

En cuanto a las características de las 22 mujeres que tuvieron abortos provocados, se encontró que 18 eran mayores de 20 años, y que de las 4 adolescentes que abortaron, 2 tenían menos de 18 años; 13 no habían pasado del 6to. grado de primaria; 19 eran casadas o unidas. Entre adolescentes hubo una razón de 0.6 abortos por cien nacidos vivos, mientras que entre mujeres mayores de 20 años la cifra correspondiente era de 1.3 por cien. Mujeres de todos los niveles de escolaridad recurrieron al aborto. Información más detallada sobre las mujeres que abortaron se presenta en la Tabla 1.

Las características de las mujeres que abortan, coinciden bastante con datos de República Dominicana, México y Perú: menos del $20 \%$ son adolescentes, más del $80 \%$ son casadas o acompañadas, y más de dos tercios son mujeres que ya han tenido hijos ${ }^{[8]}$. 
Tabla 1: Características de las mujeres que reportaron haber tenido un aborto provocado en los doce meses previos a las encuestas. Nicaragua, 1997-2005.

\begin{tabular}{|c|c|}
\hline Características & Número \\
\hline \multicolumn{2}{|l|}{ Edad } \\
\hline $15-19$ & 4 \\
\hline $20-29$ & 7 \\
\hline $30-39$ & 8 \\
\hline$\geq 40$ & 3 \\
\hline \multicolumn{2}{|l|}{ Escolaridad } \\
\hline Ninguna & 5 \\
\hline $1-3$ primaria & 3 \\
\hline 4-6 primaria & 5 \\
\hline Secundaria & 6 \\
\hline Universitaria & 3 \\
\hline \multicolumn{2}{|c|}{ Situación conyugal } \\
\hline Casadas o unidas & 19 \\
\hline Separadas & 2 \\
\hline Solteras & 1 \\
\hline \multicolumn{2}{|l|}{ Paridad } \\
\hline Sin hijos & 5 \\
\hline Con hijos & 17 \\
\hline Total & 22 \\
\hline
\end{tabular}

\section{CONCLUSIONES}

Se obtuvo una frecuencia de 1.3 abortos inducidos al año, por cada mil mujeres en edad fértil, y una razón de 1.23 abortos por cada 100 nacidos vivos. Estas cifras deben de considerarse como el mínimo de lo que ocurre en la realidad, ya que mediante otros procedimientos, principalmente a través de los métodos indirectos, se estiman cifras bastante mayores.

La frecuencia era mayor en la capital del país. Predomina en mujeres adultas, en casadas o acompañadas y en mujeres que ya han tenido hijos. Mujeres de todos los niveles educativos recurren al aborto.

\section{AGRADECIMIENTOS}

Nuestro profundo agradecimiento a las mujeres entrevistadas, al equipo de investigación, y al proyecto PROSIM-GTZ, que financió las encuestas.

\section{BIBLIOGRAFIA}

1. Ministerio de Salud y UNICEF. Mortalidad materna: un estudio de las muertes ocurridas en Nicaragua en (1998). Ministerio de Salud, Managua, Nicaragua, p. 11.

2. Padilla K, McNaughton, H. (2003). La mortalidad materna en Nicaragua: una mirada rápida a los años 2000-2002. Ipas Centroamérica. Managua, Nicaragua.

3. Peña R., Zelaya E., Liljestrand J., Dahlblom K. y Person. La Salud reproductiva e infantil en León. Octubre-Diciembre 1993. Departamento de Medicina Preventiva, Universidad Nacional Autónoma de Nicaragua, León, y Departamento de Epidemiología y Salud Pública, Universidad de Umea, Suecia.

4. Olinto MT y Moreira D., (2004). Estimating the frequency of induced abortion: a comparison of two methods. Rev. Panam. De Salud Pública, vol. 15, No. 5, p. 331-336.

5. Ahman E. and Shah Iqbal., (1999). Unsafe abortion: global and regional estimates of the incidence of unsafe abortion and associated mortality in 2000. World Health Organization, Fourth edition. (NLM clasification: WQ 440).

6. Henshaw S., Singh S. y Haas T., (1999). La incidencia del aborto inducido a nivel mundial. Perspectivas Internacionales en Planificación Familiar, p. 16-24.

7. Remez Lisa, (1995). Confrontando la realidad del aborto en América Latina. Perspectivas Internacionales en Planificación Familiar, p. 29-33.

8. Bankole A., Singh S. and Taylor H. (1999). Characteristics of women who obtain induced abortion: a worldwide review. International Familiy Perspectives, vol. 25, p. 68-77. 